Trend analysis showed participants report improvements in confidence that were equivalent between the two courses. Thematic analysis demonstrated clear constructive alignment between learning outcomes, course content and participant learning. Our findings indicate that communications skills training delivered via blended learning can deliver equivalent outcomes to face-to-face learning.

\section{P-53 'ARTSI' (ART FOR SELF-ISOLATION) - A LOCKDOWN EMAIL SERIES TO STAY CONNECTED}

Emily Santos, Caroline Allen. Peace Hospice, Watford, UK

\subsection{6/spcare-2021-Hospice.71}

Background Engaging in a creative process can help improve social functioning (Lefevre, Economos, Tricou, et al., 2020) and reduce symptom intensity (Collette, Guell, Farinas, et al., 2020), anxiety and depression (Bosman, Bood, Scherer-Rath, at al., 2021) for palliative individuals. The pandemic suspended all in-person creative services at two hospices, so a move to virtual support was required. An email series, 'ArtSi', was developed to continue providing creative arts support remotely.

Aims Weekly emails were designed at enhancing wellbeing through creative practice and generating a sense of connection (Jensen, Bonde, 2018). They offered a channel for patients, carers and bereaved individuals to stay present and mindful, relieve stress, play, momentarily forget distressing factors and find relief from overwhelming emotions.

Method Each Monday, a creative prompt/activity was emailed to $280+$ individuals who the hospices support. Participants were encouraged to reply with a photo of their completed artwork by the following Sunday, so everyone's creations could be shared together in the next week's email - creating a sense of connection and community.

Results The email series spanned over 20 weeks and we received $500+$ artworks from individuals (emailed and physical). Participants reported that they looked forward to receiving the new email each Monday as it often provided some light on darker days and a way to find pleasure and healing through enjoyment, creation and communication.

Conclusion The ArtSi emails were successful in keeping us in touch with those who we usually support through the arts, and highlighted the positive influence that creative practice and reflection can have on an individual's life (Stuckey \& Nobel, 2010). The email series provided a means for continued engagement and connection (especially for those who were particularly vulnerable or socially isolated) throughout the difficult and uncertain lockdown period.

'ArtSi has really kept me sane during lockdown. Once the art journaling sessions at the hospice had to end, I wasn't sure how to go forward... ArtSi and keeping creative have been a real life-saver.'

\section{P-54 CREATING AN INFORMATION HUB TO SUPPORT PEOPLE LIVING WITH TERMINAL ILLNESS DURING A GLOBAL PANDEMIC}

Matthew Williams. Marie Curie, London, United Kingdom

10.1136/spcare-2021-Hospice.72
Background When the UK went into national lockdown in March 2020, there were many questions about what that would mean for someone with a terminal illness. At a time when many face-to-face services had to stop, people had more questions than ever before. There was little government guidance for specific questions related to terminal illness and caring. Many carers, family members and people with an illness felt more isolated than ever before.

Aim We wanted to make a dedicated online information hub to provide a place where these questions could be answered, linking to our wider support offer.

Method We streamlined our usual content production process to get this information to our audience at the time they needed it. This allowed a small team to continually update our pages in plain English, providing guidance for all four nations of the UK as rules regularly changed. We maintained a high level of involvement from health and social care professionals, as well as policy stakeholders, ensuring the content was accurate, up-to-date, and relevant for our audience. The hub is available free on our website, and consists of 23 pages. The comprehensive resource answers questions related to coronavirus (and restrictions) for those at different stages of the caring journey - it is not just aimed at people who have (or had) COVID-19.

Results We monitored impact using Google Analytics and page surveys. Between 1 April 2020 and 31 March 2021, the hub had been accessed by 879,653 users. The page views for the hub were regularly between $25 \mathrm{k}-30 \mathrm{k}$ per week during this period. Three quarters of our users found the page they were reading useful, and said they felt better informed and reassured. The resource was shortlisted (final five from over 260 entries) for the BMA Patient Information award in 2021.

\section{P-55 DEVELOPING A 'KEEP CONNECTED' HELPLINE DURING THE COVID-19 PANDEMIC}

Jane Hazeldine. Mountbatten, Isle of Wight, UK

\subsection{6/spcare-2021-Hospice.73}

The COVID-19 pandemic had significant impact on how services, such as the hospice social programme and bereavement groups, were able to operate; many were either suspended or had to move to an online model. Understandably this affected individuals who relied on that social contact to manage and cope with their well-being and grief. In recognition of this Mountbatten created a helpline for all individuals living on the Isle of Wight affected by isolation, loneliness and bereavement. The 24/7 'Keep Connected Helpline' was developed in March 2020 and was supported by both staff and volunteers. Mountbatten recruited volunteers with the willingness and skills to manage the helpline. All volunteers received comprehensive training to respond to sometimes very complex calls. They received regular support and supervision from trained staff.

Outcomes The numbers of calls received were low but these calls were often complex. It was clear that some individuals with mental health needs were finding it hard to manage in the lockdown, and many bereaved individuals were struggling to cope with their grief in the absence of their usual peer and social network. One call was received 